\title{
Lateral Organization, Bilayer Asymmetry, and Inter-Leaflet Coupling of Biological Membranes
}

\author{
Jonathan D. Nickels ${ }^{1,2}$, Jeremy C. Smith ${ }^{3,4}$, Xiaolin Cheng ${ }^{3,4^{*}}$ \\ ${ }^{1}$ Joint Institute for Neutron Sciences, Oak Ridge National Laboratory, Oak Ridge, TN, USA \\ ${ }^{2}$ Department of Physics and Astronomy, University of Tennessee, Knoxville, TN, USA \\ ${ }^{3}$ Center for Molecular Biophysics, Oak Ridge National Laboratory, Oak Ridge, TN, USA \\ ${ }^{4}$ Department of Biochemistry and Cellular and Molecular Biology, University of Tennessee, Knoxville \\ TN, USA
}

*Correspondence to:

Xiaolin Cheng

Oak Ridge National Laboratory

P.O. Box 2008, MS-6309

Oak Ridge, TN 37831, USA

Email: chengx@ornl.gov 


\begin{abstract}
Understanding of cell membrane organization has evolved significantly from the classic fluid mosaic model. It is now recognized that biological membranes are highly organized structures, with differences in lipid compositions between inner and outer leaflets and in lateral structures within the bilayer plane, known as lipid rafts. These organizing principles are important for protein localization and function as well as cellular signaling. However, the mechanisms and biophysical basis of lipid raft formation, structure, dynamics and function are not clearly understood. One key question, which we focus on in this review, is how lateral organization and leaflet compositional asymmetry are coupled. Detailed information elucidating this question has been sparse because of the small size and transient nature of rafts and the experimental challenges in constructing asymmetric bilayers. Resolving this mystery will require advances in both experimentation and modeling. We discuss here the preparation of model systems along with experimental and computational approaches that have been applied in efforts to address this key question in membrane biology. We seek to place recent and future advances in experimental and computational techniques in context, providing insight into in-plane and transverse organization of biological membranes.
\end{abstract}




\section{Introduction}

The lipid raft hypothesis has fundamentally changed the way we look at the cell membrane and how it organizes proteins and lipids to accomplish its varied and vital functions ${ }^{1}$. Through the selective partitioning of membrane proteins between rafts and their surroundings, rafts control protein-protein interactions, enhancing certain associations while suppressing others. In this manner, rafts are thought to mediate a range of cellular processes, including signal transduction ${ }^{2}$, apoptosis ${ }^{3}$, cell adhesion and migration $^{4}$, cell recognition ${ }^{5}$, synaptic transmission ${ }^{6}$, cytoskeletal organization ${ }^{7}$ and protein sorting ${ }^{8,9}$. The rafts themselves are thought to be small $(10-100 \mathrm{~nm}){ }^{10}$, heterogeneous ${ }^{11}$ and highly dynamic ${ }^{12}$ structures composed primarily of lipids but also rich in sterols, carbohydrates, and proteins ${ }^{13}$. Rafts have been extensively studied in animal cells ${ }^{14,15}$, and recent research has shown that they are also central to the organization and function of membranes in plants ${ }^{16}$ and microbes ${ }^{17}$. They also play a role in bacterial and viral pathogenesis ${ }^{18,19}$. The size, lifetime, and connectivity of rafts are crucial parameters for understanding their function ${ }^{8}$. How these parameters are controlled by the cell is, at best, poorly understood and is the focus of intense current scientific interest.

Another key feature of biological membranes is that they are compositionally asymmetric ${ }^{20}$. In fact, bilayer compositional asymmetry was well known even within five years ${ }^{14,21-26}$ of the publication of the fluid mosaic model ${ }^{27}$. The majority of aminophospholipids reside on the membrane inner leaflet, with sphingomyelin and choline phospholipids primarily in the outer leaflet (Fig.1) ${ }^{14,24}$. Cholesterol is found in both bilayer leaflets, but distributed asymmetrically, with a greater concentration suggested for the inner leaflet ${ }^{28}$. This asymmetry impacts a range of bilayer properties ${ }^{29-31}$ and is tied to numerous biological functions, including signaling of apoptosis ${ }^{32-35}$, thrombosis ${ }^{25,36}$, phagocytosis ${ }^{32,37-39}$, and as an indicator of tumorigenic cells ${ }^{40,41}$. In vivo, bilayer asymmetry is maintained by adenosine triphosphate (ATP)-dependent enzymes that transport lipids between the two leaflets ${ }^{42,}{ }^{43}$. However, generation of asymmetry in experimentally accessible model systems has, until very recently, proven elusive ${ }^{44-47}$ meaning that most work on membrane biophysics has made use of symmetric self-assembled bilayers.

In the context of membrane domains, the absence of asymmetry has been recognized as a significant deficiency ${ }^{45,46,48}$ and leaves a number of important questions unanswered. Key among these is the role of inter-leaflet coupling (Fig. 2) in formation of lipid rafts. Current consensus is that domain formation in the membrane outer leaflet is coupled to the inner leaflet, and this interdependence is important for both the structure of the membrane and the communication of information across it ${ }^{45,46,49}$. It is known that symmetric model membranes made from lipid mixtures emulating the outer leaflet of mammalian cells exhibit nanoscopic phase behavior characteristic of lipid rafts. However, lipid mixtures emulating the inner leaflet fail to do so, remaining uniformly mixed ${ }^{50,51}$. This is intriguing because rafts are expected to exist in both leaflets of the bilayer ${ }^{49,52,53}$, implying a critical role of inter-leaflet coupling in vivo. Moreover, the mechanisms by which the outer leaflet of the bilayer could interact with the inner leaflet remain unknown. 


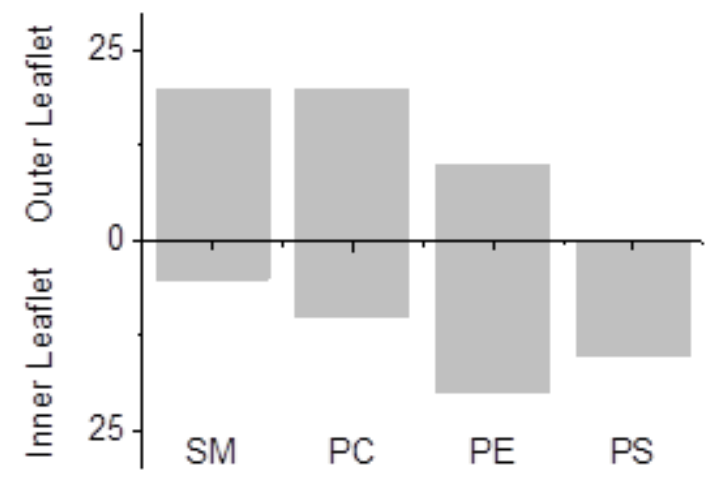

Figure 1 . The cell membrane of human red blood cells possess an asymmetric distribution of lipids $(\mathrm{SM}=$ sphingomyelin, $\mathrm{PC}=$ phosphatidylcholine, $\mathrm{PE}=$ phosphatidylethanolamine and $\mathrm{PS}=$ phosphatidylserine) between the inner leaflet (cytoplasmic) and outer leaflet (extracellular) ${ }^{14}$.

Perhaps the most intuitive explanation for interleaflet coupling in cell membranes is the presence of membrane-spanning proteins that physically bridge the two bilayer leaflets. However, observations in protein-free model systems suggest that the coupling may be an intrinsic property of the lipids themselves ${ }^{30}$. Experimental work has shown direct evidence of interleaflet coupling in the form of domain alignment between the bilayer leaflets in symmetric giant unilamellar vesicles (GUVs) and supported bilayers ${ }^{51,54-57}$. An alternative coupling mechanism has also been reported, manifested as anti-correlation in small (nm) or solid domains ${ }^{58-62}$. Owing to recent advance in generating asymmetric vesicle models, calorimetric measurements of melting transitions in these asymmetric models also supported the notion of interleaflet coupling ${ }^{63}$. A number of excellent reviews on inter-leaflet domain coupling have been written ${ }^{44-46}$. The present review will focus on bringing this topic together with recent developments in three areas: generating asymmetric model bilayers, neutron scattering methods, and computer simulation techniques. Neutron scattering and computational methods are naturally compatible techniques, able to detect and analyze nanoscopic domains and lipid asymmetry. Together with recent developments in generating model asymmetric bilayers there is a tremendous opportunity to offer new insights into the mechanisms of raft formation and interleaflet coupling.
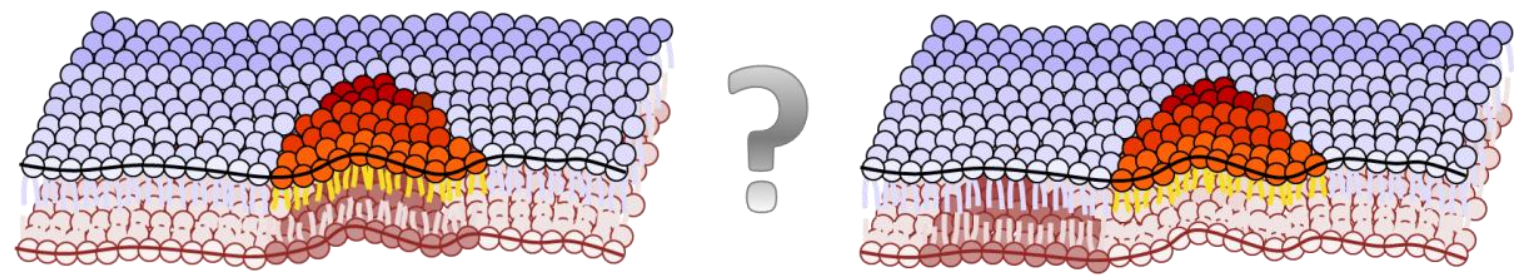

Figure 2. Key outstanding questions in membrane biophysics center on the interplay of leaflet compositional asymmetry and lateral organization. Are lipid rafts 'in-registry"? Can leaflet coupling induce raft formation in the mammalian inner leaflet?

\section{Experimental studies of cross-layer coupling}




\subsection{Preparation of asymmetric bilayers}

Replicating the asymmetry of biological membranes is a critical starting point for studies of bilayer coupling. One might consider harvesting naturally occurring lipid bilayers in order to take advantage of the asymmetry native to biological membranes. In fact, Weik and coworkers have used neutron scattering on crystalline membrane structures known as purple membranes to demonstrate the asymmetric bilayer orientation of glycolipids with respect to membrane proteins ${ }^{207}$. Unfortunately, most natural systems are non-crystalline, and moreover natural constructs typically lose asymmetry over time. It is advantageous then to produce model systems which replicate the asymmetry of biological membranes. Methods for generating asymmetric model bilayer systems have progressed in recent years, using passive mechanisms (cyclodextrin exchange, Langmuir-Blodgett), emulsification strategies (microfluidics), or active mechanisms (lipid flippase/floppase enzymes).

Supported lipid bilayers Supported lipid bilayers are classic models for biological membranes. They allow membrane constituents to be studied in a controlled environment, and are compatible with many experimental probes, such as fluorescence microscopy, atomic force microscopy and neutron reflectometry. This control comes at a price however, with the substrate influencing the bilayer, reducing hydration and introducing artifacts to the structure and dynamics of the lipids.

One of the most convenient ways of preparing supported bilayers is vesicle fusion to a substrate. Since the pioneering work of McConnell and co-workers ${ }^{64}$, supported bilayers have been formed by vesicle fusion on various substrates, such as glass, mica, self-assembled mono-layers, polymers, and quartz. Asymmetric supported bilayers can be prepared by sequential deposition of lipid monolayers using the LangmuirBlodgett/Langmuir-Schafer (LB/LS) or the Langmuir-Blodgett/vesicle fusion (LB/VF) methods. Care must be taken with these approaches, as the asymmetric bilayers prepared this way often do not have the intended lipid compositions in the two leaflets. Because of this, two additional techniques are critical for the characterization and production of asymmetric supported bilayers ${ }^{44}$. The first is the use of the fluorescence interference contrast (FLIC) microscopy ${ }^{65}$ to measure lipid flip-flop across supported membranes. In this way, the compositional asymmetry can be quantified. The second is the development of the tethered polymer supported bilayer ${ }^{66}$. It has proven to be critical for maintaining bilayer asymmetry to add an inert, highly hydrated polymer cushion between the membrane and the solid substrate.

Domain formation has been studied using supported bilayers, providing an important example of interleaflet domain induction ${ }^{44,65,67}$. Tamm and coworkers have studied the PC/PE/PS/cholesterol mixtures extracted from natural brain or egg extracts that mimic the cytoplasmic leaflet of cell membranes ${ }^{44}$. These mixtures cannot form phase-separated domains in symmetric bilayers. However, this composition was induced to form domains when opposing a $\mathrm{PC} / \mathrm{SM} /$ cholesterol leaflet that mimics the exoplasmic leaflet of cell membranes. It was also concluded that at least one high melting temperature, $T_{m}$, lipid, one low $T_{m}$ lipid and cholesterol need to be present for domain formation in the second leaflet by coupling to preexisting domains in the first exoplasmic leaflet ${ }^{68}$.

Unilamellar vesicles Unilamellar vesicles (ULVs) are important structures that resemble biological membranes far more than supported bilayers. In addition to mimicking biological membranes in biophysical studies, lipid vesicles are used to entrap proteins molecules ${ }^{69,70}$ or drug delivery ${ }^{71,72}$ and even act potential blood substitutes ${ }^{73-76}$. The unsupported bilayer of a ULV has more physiologically relevant hydration, natural boundary conditions, and enables facile incorporation of membrane proteins. ULVs also offer flexibility in terms of size (or curvature). Small unilamellar vesicles are ideal for mimicking highly curved membranes (endosomes ${ }^{77}$, synaptic vesicles ${ }^{78}$, etc.), whereas giant unilamellar vesicles are suitable for very low curved membranes (the cell plasma membranes). It should be noted however, that highly curved bilayers can be considered asymmetric due to geometric packing effects ${ }^{79}$, complicating coupling studies. ULVs have become one of the most widely applicable membrane models and have been 
used in a greater variety of spectroscopic and biochemical studies, such as small angle neutron scattering (SANS) and neutron spin echo (NSE) experiments.
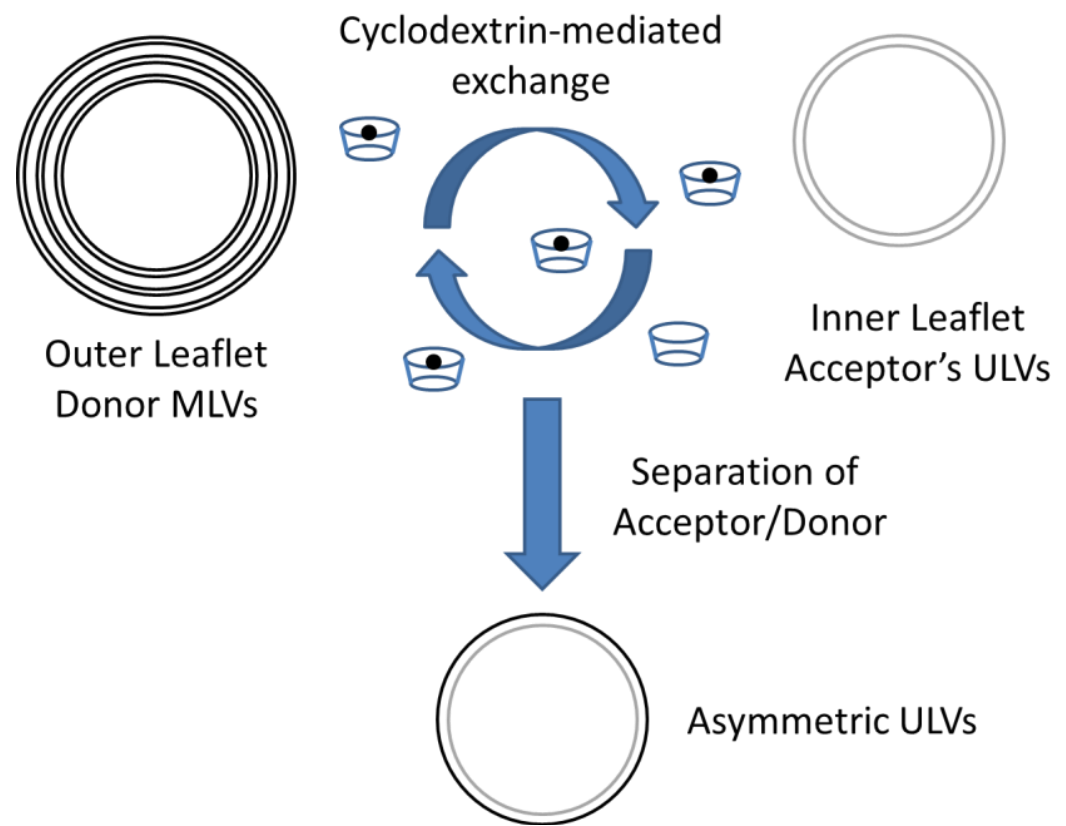

Figure 3. Schematic of asymmetric vesicle production through cyclodextrin mediated exchange ${ }^{29}$.

Symmetric ULVs are typically prepared by sonication or extrusion through polycarbonate filters of defined size. To incorporate compositional bilayer asymmetry, London and co-workers developed a technique, in which cyclodextrin-mediated lipid exchange is used to prepare ULVs with different compositions across the lipid bilayers (Figure 3). ${ }^{29}$ In this method, two populations of vesicles are prepared, ULVs with the desired inner leaflet composition, and MLV with the desired outer leaflet composition. These two populations are mixed and incubated with cyclodextrin. The choice of cyclodextrin depends on the presence or lack of cholesterol, with methyl- $\beta$-cyclodextrin useful for pure lipid systems ${ }^{29,30,63,80}$ and hydroxypropyl- $\alpha$-cyclodextrin used when cholesterol is present in the acceptor ULVs ${ }^{80-83}$. This approach has proven to be flexible, producing model systems that closely mimic the mammalian plasma membranes (SM and/or PC outside and PE/PS inside), and in which cholesterol content can be readily varied between 0 and $50 \mathrm{~mol} \%$. These asymmetric ULVs should represent more realistic model membranes for probing phase behaviors as well as the interplay between membrane asymmetry and interleaflet domain coupling.

Emulsion/microfluidic approaches There have also been significant advances in microfluidic technologies in recent years ${ }^{84}$. This has allowed researchers to controllably generate monodisperse lipid vesicles using a double emulsion approach ${ }^{85,86}$. In this method, a water-in-organic emulsion droplet is formed, stabilized by what is to become the inner leaflet monolayer. These droplets, and the surrounding organic solvent, are then immersed into an additional aqueous phase with a second supported interfacial layer (the outer bilayer leaflet) to form a water-in-organic-in-water double emulsion. Upon removal of the organic phase, this method generates monodisperse bilayers with controllable bilayer composition. Using these microfluidic approaches, several recent studies have generated asymmetric vesicles starting from two different lipids or lipid mixtures ${ }^{87-91}$.

A final comment about asymmetric ULVs and supported bilayers is that these systems are not in equilibrium. The lipids in each bilayer leaflet are known to spontaneously exchange ${ }^{92}$ in a process called 'flip-flop', reducing any imposed asymmetry as a function of time. This 'flip-flop' rate is a feature of the 
state of the lipids themselves, as well as their acyl chain length, the presence of ions in the buffer and/or supporting interfaces ${ }^{93-97}$.

Protocell systems Biology has solved the technical problem of creating stable asymmetric bilayers and maintaining them, through a series of ATP-dependent enzymes known as flippases, floppases, and scramblases ${ }^{42,45,98,99}$. In this regard, a protocell containing some of these enzymes is an attractive concept for developing asymmetric lipid vesicles that are suitable for characterizing its transverse and lateral structures in a realistic manner. This is somewhat different from most research efforts related to protocell systems that center on self-replication and artificial life ${ }^{100}$. Protocell systems will also provide a versatile platform for assessing protein activity in its native-like environments (i.e. different lipid composition), and for revealing the correlation between protein structure/function and phase state of the membrane.

The basic approach of the protocell utilizes ULVs containing a well-defined binary mixture. Membrane proteins (flippases for instance) can be reconstituted in ULVs using one of two common approaches: 1) the dilution of the protein-detergent mixture with ULV solutions, or 2) the introduction of detergents to preformed ULVs followed by addition of the protein-detergent mixture. Asymmetric distributions of phospholipids between the cytoplasmic and exoplasmic membrane leaflets in biological cells are usually maintained by flippases ${ }^{101}$, a family of transmembrane lipid transporter enzymes responsible for the movement of phospholipid molecules from one leaflet to the other. Therefore, through incorporation of the flippases, membrane asymmetry will be generated and maintained by active transport of phospholipids across the protocell membrane leaflets. Active transport means that energy input in the form of ATP is required by the flippases to transfer phospholipid molecules in a specific direction, i.e., from the cytoplasmic face to the exoplasmic face of the membrane. When coupled with a minimum ATP generation system ${ }^{102}$, the protocell will be able to maintain membrane asymmetry between the two membrane leaflets in a self-contained manner. For optimal functional studies, the developed protocell should fulfill a number of criteria, such as uniform distribution of proteins and their proper orientation (and activity whenever it is possible) in the bilayer. The protocell system provides an active approach for generating and maintaining membrane asymmetry, enabling a deeper understanding of membrane composition, the interplay between compositional asymmetry and lipid rafts, as well as the function of ATP-driven membrane proteins in a physiologically relevant membrane environment.

\subsection{Methods to Study Lipid Organization}

Many experimental methods have been used to study the transverse structure of lipid bilayers. Lipid asymmetry was primarily determined using fluorescent ${ }^{103,104}$, radiochemical $^{21,26}$, and spin labeling (ESR) $42,{ }^{105}$, as well as nuclear magnetic resonance (NMR) ${ }^{26}$. More recently, atomic force microscopy (AFM) 106 has been used to study lipid asymmetry in supported bilayer systems. Fluorescence interference contrast microscopy (FLIC) ${ }^{65}$, total internal reflection fluorescence microscopy (TIRF) ${ }^{107,}{ }^{108}$ and fluorescence correlation spectroscopy $(\mathrm{FCS})^{109}$ are a few of the other recent developments useful to quantify asymmetry in supported systems.

Detection and interrogation of lipid rafts, lipid domains, and other lateral heterogeneities present a different challenge. Detergent resistance ${ }^{110}$ and cyclodextrin depletion ${ }^{111}$ were key early methods used to study lipid raft phenomena while $\mathrm{AFM}^{112-114}$ and fluorescence microscopy (FM) ${ }^{115}$ have emerged as more recent techniques to characterize lateral organization ${ }^{116}$. AFM provides detailed information about bilayer thickness but is limited to model or isolated native membranes that can be immobilized on surfaces. FM is able to interrogate domain size, shape and physical properties ${ }^{117}$ based on the partitioning of dye molecules into the different phases ${ }^{118}$ but is limited by the diffraction limit. Single molecule ${ }^{119}$, multiphoton methods ${ }^{120}$ and a range of super-resolution microscopy techniques ${ }^{121}$ are being developed in efforts to overcome this. Förster resonance energy transfer (FRET) ${ }^{122}$ is able to probe much smaller distances and has proven extremely useful for studying domain formation and phase behavior ${ }^{123,}{ }^{124}$ as 
well as identifying raft associating molecules ${ }^{125}$. FM has been also been used to study partitioning, diffusion and ligand binding of raft lipid analogs in model and cellular plasma membranes ${ }^{126}$. Fluorescence recovery after photobleaching (FRAP) ${ }^{127}$ is a particularly useful approach for studying lateral diffusion in bilayers ${ }^{128}$ and distinguishing the liquid ordered (Lo) phase in raft forming models ${ }^{129}$, ${ }^{130}$. It works by photo-bleaching a defined area and measuring the rate at which fluorophores diffuse back into the bleached spot.

The above methods have provided, and will continue to provide, useful tools for the study of lipid organization. Yet, they have a number of important limitations to keep in mind, such as the potential perturbations of molecular probes or surfaces, and the size of some lipid domains (especially those of biological relevance) being smaller than the detection capabilities of optical microscopy. We will now discuss other scattering methods that can probe free floating and label free bilayer systems.

\subsection{Small angle neutron scattering (SANS)}

Small-angle neutron scattering (SANS) is an experimental scattering technique which measures the elastic scattering of neutrons from atomic nuclei to probe structures from $\sim 1$ to 100 nanometers. SANS is uniquely useful in studies of biological materials due to its sensitivity to light elements, particularly hydrogen and its isotope deuterium. In fact, some of the early studies of the transverse structure of the lipid bilayer used neutrons and isotopic substitution of the various components of the lipid molecule ${ }^{131-134}$. Typically, in structural studies of lipids, the scattering of the material is quantified through the neutron scattering length density (NSLD) which reflects the average scattering length of the atoms within a given volume. This is regulated through the deuterium/hydrogen ratio of the solvent and/or lipid. More recently, SANS is emerging as a powerful tool for studying the lateral structures of lipid membranes ${ }^{135-137}$.
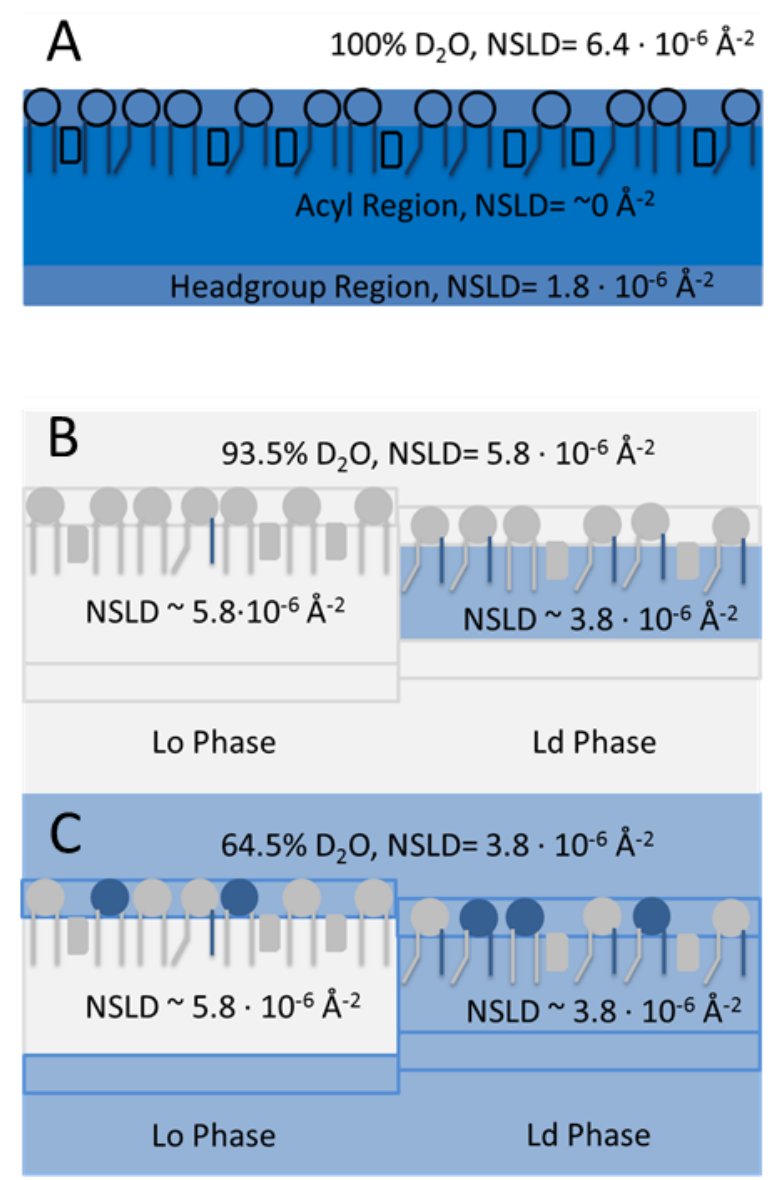
Figure 4. An alternative contrast matching scheme used by Nickels et al. ${ }^{53}$ to measure individual phases within phase separated lipid bilayers composed of POPC/DSPC/Chol. A) Structure and NSLD of a hydrogenated bilayer in $\mathrm{D}_{2} \mathrm{O}$. B) and C) show the NSLDs and schemes used to isolate scattering from the acyl region of individual lipid phases.

These advances make use of the fact that SANS allows for the scattering from individual bilayer components to be suppressed independently through the practice of NSLD contrast matching techniques. Scattering intensity scales with the square of the difference in NSLD between adjacent materials within the sample (Fig. 4). By making that difference equal to zero, all scattering from a given structure is suppressed, isolating the scattering from unmatched regions. Isotopic substitutions can be used to match the NSLD of particular structural units to each other or the solvent. In this way, an asymmetric leaflet or lipid domain could have a different NSLD than the rest of the system, providing unique access to this structural information. Neutron scattering is also an effectively probe-free technique that introduces minimal perturbation to membrane composition. This is particularly advantageous for systems that are sensitive to the presence of extrinsic probes (e.g., bulky fluorophores) or studies of phase behavior, where compositional variations are critical.

Detection of nanoscopic domains in membranes by SANS Katsaras and co-workers have driven the use of SANS in the detection of lipid domains. This effort began with Pencer and co-workers ${ }^{135}$ describing a set of measurements designed to detect lateral heterogeneity within lipid bilayers using SANS. Their experiment was based on the idea that the SANS signal that arises from laterally phase separated ULVs can be described using three components: (i) a component reflecting the NSLD contrast between the average bilayer composition and the solvent; (ii) a radial component from NSLD variation in the direction normal to the bilayer plane (which can be approximated by slabs corresponding to the headgroup and acyl chain regions); and (iii) a lateral component from NSLD variation within the plane of the bilayer. They predicted that if one took a lipid mixture which phase separates above the temperature where the phases mix, resulting in homogenous mixing of the lipid components, it would eliminate the lateral scattering contribution, allowing for the detection of domains, shown schematically in Fig. 5.

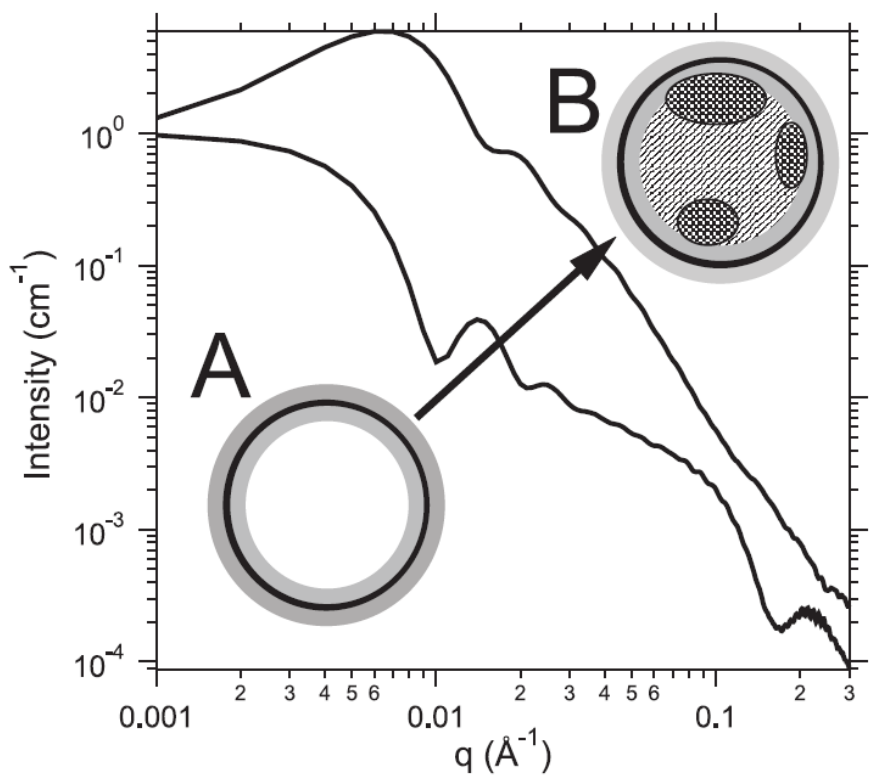

Figure 5. Scheme for SANS scattering from phase separated lipid vesicles. Excess scattering from lateral organization is emphasized from the comparison of scattering data below (A) and above (B) the mixing transition. Figure adapted from Pencer et al. ${ }^{135}$ 
With detailed knowledge of a lipid phase diagram and deuterated materials, this scheme can be improved by matching the SLD of homogenously mixed lipid components to that of the solvent. Both the solvent and lipid NSLDs can be tuned experimentally by adjusting the ratio of $\mathrm{D}_{2} \mathrm{O} / \mathrm{H}_{2} \mathrm{O}$ in the aqueous medium, and the ratio of deuterated/protiated chains in the bilayer. Heberle et al. adopted this approach in SANS experiments using a four-component model system containing fixed proportions of cholesterol and the saturated phospholipid 1,2-distearoyl-sn-glycero-3-phosphocholine (DSPC), mixed with varying amounts of the unsaturated phospholipids 1-palmitoyl-2-oleoyl-sn-glycero-3-phosphocholine (POPC) and 1,2dioleoylsn-glycero-3-phosphocholine (DOPC) ${ }^{136}$. By matching the average NSLD of the solvent, headgroup and acyl chain region, they were able to observe excess scattering signal arising only from the lateral segregation in the acyl chain region upon domain formation. Through systematic changes in the composition, a direct correlation was found to exist between the domain size and the mismatch in bilayer thickness of the coexisting liquid ordered (Lo) and liquid disordered (Ld) phases, suggesting a dominant role for the interfacial energy between the domains in controlling domain size.

Recent work has further developed this idea ${ }^{53}$, by altering the contrast strategy again to focus on matching individual phases within a vesicle. By matching the NSLD of the solvent to all of the head groups and the acyl region of one phase, it is possible to isolate the acyl chains of the other phase, as depicted in Fig 4. Studying the scattering of individual phases provides a range of new possibilities. For example, by examining the acyl thickness of individual phases, it was determined that nanodomains in the POPC/DSPC/cholesterol system are in register, or aligned across the two bilayer leaflets ${ }^{53}$. For antiregistered phases, one would expect to see only a monolayer thickness due to the apposition of visible Ld phase and the invisible Lo phase. This NSLD contrast matching scheme also enables neutron scattering measurements to study dynamics of individual phases. NSE measurements are sensitive to the thermal undulations of the bilayer, allowing one to extract the bending modulus ${ }^{138}$. It was shown that the nanoscopic domains were dynamically decoupled from the surrounding phase, showing that they maintain bending properties similar to the Ld composition studied in bulk. This has implications for domain formation and stability, and highlights how neutrons are ideal for the study of lipid bilayer organization.

Potential of SANS Measurements for Lipid Asymmetry. Given recent developments in the generation of asymmetric lipid vesicles that we described above, we can begin to consider how neutrons will be optimally deployed to study their structure and properties. The most straightforward approach appears to introduce isotopic differences between the two bilayer leaflets using the methods of London and coworkers $^{29}$. Extending this to deuterated materials for use in scattering experiments is a logical next step, but it should be noted that deuterated materials are denser than their hydrogenated counterparts, which complicates the production of asymmetric vesicles, requiring changes in the protocols for separation of donor and acceptor species. If this issue is solved though, this would allow for neutron reflectivity measurements on supported asymmetric lipid bilayers or SANS measurements on free floating vesicles. In Fig. 6, we present a hypothetical SANS experiment on $50 \mathrm{~nm}$ radius asymmetric vesicles where one leaflet contains deuterated acyl chains. There is a clear effect of asymmetry on the scattering patterns that reflects the changes in the transverse NSLD profile, but the inner versus outer leaflet exchanged vesicles result in similar spectra. This is the case of an ideal asymmetry, but real experiments would be subject to a number of other effects that would actually make the result more informative. Inefficiencies in lipid exchange, flip-flop in the course of the measurements, and geometric asymmetry would influence the leaflet thicknesses and NSLD, making the two cases of inner and outer leaflet deuteration clearly distinguishable. 


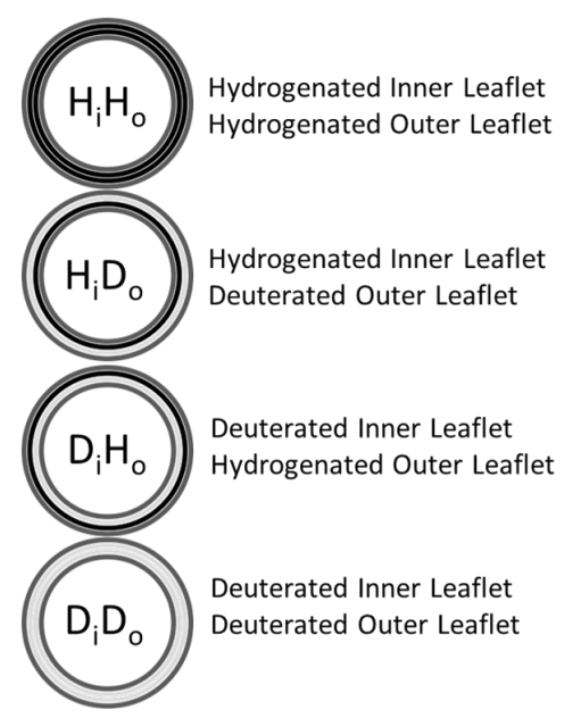

Basic 'slab' model for scatting from $50 \mathrm{~nm}$ radius asymmetric vesicles:

$I(q)=A\left[\sum_{i=1}^{5} \frac{3 V_{i}\left(\rho_{i}-\rho_{i-1}\right) j_{1}\left(q d_{i}\right)}{q d_{i}}\right]^{2} S(q)+b k g$

Here, $\rho_{i}$ and $d_{i}$ define the NSLD and distance from the vesicle center for each slab. $A$ is the scale factor, $b k g$ is the constant background, $V_{i}$ is the volume of each shell, $j_{1}$ is the Bessel function. $S(q)$ is the structure factor which reduces to 1 for dilute vesicle suspensions.
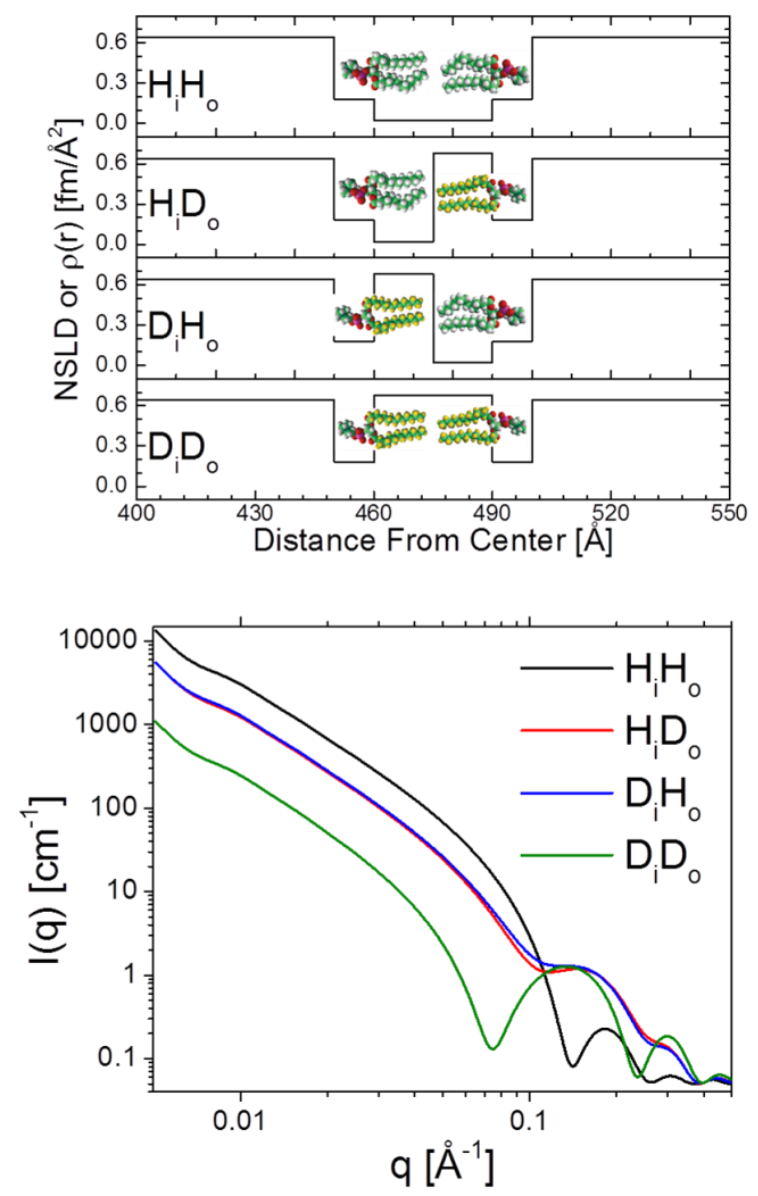

Figure 6. Bilayer asymmetry can be experimentally observed by SANS using selective deuteration of individual bilayer components. For instance, if one deuterates the hydrocarbon chains in one leaflet, the resulting scattering can be predicted using a traditional slab model ${ }^{139-142}$. Here we present a model of scattering from $50 \mathrm{~nm}$ radius vesicles with a Gaussian size distribution and the SLD profiles shown in the top right panel, we also assume the instrumental resolution of the BioSANS spectrometer at HFIR, ORNL.

The above basic example with a single lipid is useful to understand the possibilities of neutrons and asymmetry, but the true impact will be realized only once this concept is developed, demonstrated, and combined with more biologically relevant asymmetric membrane models. Careful compositional control across the two bilayer leaflets opens up the possibility of using neutrons to study questions of leaflet coupling and its interplay with lateral organization. London and co-workers have already demonstrated this technical achievement with lipid only systems, cholesterol containing bilayers, and membrane mimetic mixtures of hydrogenated lipids as described above. If the technical issues around the use of deuterated materials are solved (changes in mass and density complicating the separation stage), and sufficient compositional control achieved, one could return to the advanced contrast matching approaches that found success in the study of lateral organization. This will open opportunities for a range of studies focused on questions of leaflet coupling and induced domain formation. These potential studies are presented as possible approaches that fully synthesize current capabilities from the preparation of asymmetric vesicles and scattering techniques. 


\section{Computational studies of cross-layer coupling}

In recent years, it has become apparent that the combination of molecular dynamics (MD) simulation, which uses Newtonian mechanics to compute how each atom in a system moves with time, with various experiments (such as, neutron scattering, NMR) has the potential to evolve into the definitive tool for elucidating the atomistic details of the structure and dynamics of intrinsically heterogeneous biomembranes. With advances in computer hardware and software, biomembrane simulations have become more biologically relevant in both time and length scales. Niemela et al. employed extensive allatom MD (AAMD) simulations to elucidate the structural and dynamical properties of ternary raft mixtures with cholesterol, palmitoylsphingomyelin (PSM), and POPC, providing evidence that the presence of PSM and cholesterol in raft-like membranes leads to strongly packed and rigid bilayers ${ }^{143}$. Recently, ten $\mu$ s-long AAMD simulations of a ternary mixture of DOPC, dipalmitoylphosphatidylcholine (DPPC), and cholesterol obtained on the Anton special purpose computer revealed remarkable heterogeneity in biomembranes ${ }^{144}$. Substructures were identified within the Lo phase of lipid bilayers composed of saturated hydrocarbon chains packed with local hexagonal order and separated by interstitial regions enriched in cholesterol and unsaturated chains. More recently, AAMD simulations were used to determine the biomembrane bending modulus $\left(K_{c}\right)$ based on the analysis of thermal fluctuations in lipid orientations, where the effects of lipid tilting decouple from the lipid orientation energetics associated with bending moduli ${ }^{145}$. AAMD simulation has also been combined with X-ray and neutron scattering techniques to study the structure and dynamics of pure lipid bilayers and the effects of lipid modifications

146, 147 or the addition of various small organic molecules, such as cholesterol ${ }^{146}$, carotenoids ${ }^{148}$, and tamoxifen ${ }^{149}$.

Acyl chain interdigitation has been shown to significantly affect the properties of the phase state of lipid bilayers. Niemela et al utilized AAMD simulations to study the chain interdigitation in bilayers comprised of SM species ${ }^{143}$. They quantified interdigitation of the acyl chains across the bilayer center by computing electron (and atomic number) density profiles, which revealed significant interdigitation for all acyl chain lengths considered, indicating a strong coupling of the two leaflets. In plasma membranes, the compositions of the two leaflets are highly different, and there is co-existence of Lo and Ld domains. The biological significance of interdigitation in these complex plasma membranes remains to be further explored.

However, even if using an accurate molecular mechanical (force field) model, AAMD simulations are still limited by the time and length scales that they can efficiently access. It is presently believed that the biologically relevant length and time scales of domain formation lie in the ranges of $10 \mathrm{~nm}$ to $200 \mathrm{~nm}$ and $\mu$ s to ms, respectively, both of which are 2-3 orders of magnitude beyond what can routinely be accessed by AAMD simulation. In order to examine slow processes in biomembranes, such as cholesterol flip-flop, domain formation or domain coupling between leaflets, enhanced sampling techniques must be used in conjunction with AAMD simulations. Cholesterol flip-flop motion has attracted a lot of attention since it is one of proposed mechanisms underlying the cross-layer domain coupling. Bennett et al used AAMD simulations to investigate cholesterol transfer from water to its equilibrium position in the membrane and to the center of the membrane for a systematic set of lipid bilayers, providing a detailed molecular level thermodynamic description of cholesterol interactions with lipid bilayers ${ }^{150}$. Jo et al. have explored the energetics and mechanism of passive cholesterol flip-flop and its dependence on chain saturation, by performing two-dimensional umbrella sampling simulations combined with the path-finding string method ${ }^{151}$ to identify the most probable flip-flop paths ${ }^{152}$. The calculated flip-flop rates show that cholesterol flip-flop in a poly-unsaturated bilayer is faster than in more saturated bilayers, resulting from the more favorable cholesterol-lipid interaction in polyunsaturated bilayer than in more saturated DPPC or POPC bilayers. 
AAMD simulation of the lipid mixing/demixing process (relevant to in-plane phase separation) still poses a significant challenge for most enhanced sampling techniques, since most of these methods rely on the use of a reaction coordinate (order parameter), along which the biasing forces can be applied to drive the system from one state to another. For the lipid mixing/demixing process, it is however, not trivial to identify such a geometrical order parameter that defines the phase separation path. Employing the specialpurpose machine Anton, Hong et al. performed $\mu$ s-long AAMD simulations to study the dynamics of mixing and characterize the lateral distribution of lipids in converged mixtures ${ }^{153}$. The same group also presented the first application of the accelerated molecular dynamics (aMD) method to lipid membranes. It was found that the aMD simulations produce 2-3 fold speedups in lipid lateral diffusion versus those in conventional AAMD simulations ${ }^{153,154}$. However, neither the above AAMD simulations have produced a complete phase separation from a randomly mixed lipid (and cholesterol) mixtures.

Coarse-grained MD (CGMD) has also been developed to study membrane associated molecular events that are limited by time and length scales in AAMD simulations, such as lipid mixing/demixing and cholesterol flip-flop. In CGMD, multiple atoms are grouped into a CG bead, reducing the computational cost and extending the time- and length-scales of the simulation. CGMD has been used to directly observe domain formation with molecular level detail in model membrane systems. Using the CG MARTINI force field ${ }^{155}$, Marrink and coworkers found that bilayers with compositional asymmetry were stable over a multi- $\mu$ s simulation, and they also observed the formation of registered Lo domains in a compositionally symmetric bilayer. Later on, the same group performed a series of CGMD simulations to investigate various aspects of lipid domain formation in ternary lipid mixtures of bilayers and small vesicles ${ }^{156}$. In these simulations, phase separation was shown to occur, consistent with experimentally observed Lo domains (high DPPC and cholesterol content) and Ld domains (high polyunsaturated lipid and low cholesterol content). Interleaflet domain coupling was also observed. Under the framework of elastic theory, these simulations suggested that low surface tension drives domain registration across the leaflets. Perlmutter and Sachs extended this approach, simulating interleaflet domain coupling in various asymmetric ternary lipid mixtures. They confirmed that the Lo-Ld domains coexist in three compositionally symmetric, ternary bilayers ${ }^{62}$, and further showed that domain formation in one leaflet induced ordering in the opposite leaflet composed of pure unsaturated lipids. Importantly, they also observed domain anti-registration in the asymmetric lipid mixtures with longer-chain saturated lipids, which was attributed to mismatched acyl chain-lengths in the saturated and unsaturated lipids. They concluded that the increased local lipid curvature induced by compositional asymmetry might be responsible for the interleaflet domain coupling.

Recently, Marrink and coworkers carried out large-scale MARTINI CGMD simulations, which provided a molecular view of the lipid organization of a plasma membrane at an unprecedented level of complexity 157. This plasma membrane model, closely mimicking mammalian plasma membranes, consists of 63 different lipid species, combining 14 types of headgroups and 11 types of tails, which are asymmetrically distributed across the two leaflets. The simulations showed a general non-ideal lateral mixing of the different lipid species. In particular, an enrichment of cholesterol in the outer leaflet was observed. Furthermore, the formation of low/high cholesterol density regions was found to be correlated between the two membrane leaflets, suggesting that the domains are coupled across the two leaflets even at the tens of nm length scale and on the $\mu$ s time scale ${ }^{157}$.

Besides lipid mixing/demixing simulations, CGMD simulations have also been used to directly investigate the flip-flop of cholesterol in lipid bilayers. Marrink et al showed a fast flip-flop rate for the sterol in polyunsaturated lipids along with an increased preference of cholesterol for an unusual location in the middle of the two monolayer leaflets ${ }^{158}$. Using a large set of CG simulations, Bennett et al. predicted that the rate of cholesterol flip-flop strongly depends on the composition of the bilayer. In polyunsaturated bilayers, cholesterol undergoes flip-flop on a sub- $\mu$ s time scale, while flip-flop occurs in the second range in saturated bilayers with high cholesterol content ${ }^{150}$. Fast cholesterol flip-flop in the Ld 
phase, and slow flip-flop in the Lo phase were also observed in these CGMD simulations ${ }^{156}$, implicating a potential role of flip-flop in interleaflet domain coupling.

Pantano et al. used the CGMD simulations to study the morphological phase diagram (formation of solid domains) in bilayers composed of charged di-block copolymers. They found that the charges induce a local structural rearrangement that offsets the steric repulsion between the headgroups ${ }^{159}$. They then extended the model to study domain registration in bilayers composed of mixtures of single chain polymers with identical tails but with either a charged or neutral polar headgroup. These simulations revealed domain formation, likely due to the clustering of the charged headgroups cross-linked with ions. They further quantified the interleaflet coupling strength by computing the potential of mean force (PMF) as a function of inter-leaflet domain overlap. Biasing forces were applied to move each domain in an antiparallel manner. The PMF was then estimated from the work through the Jarzynski equation ${ }^{160}$. The strength of registration can be expressed as the PMF difference between the initial (registered) and final (anti-registered) states, and this confirmed that the registered state is more stable than the anti-registered state with amplitude on the order of hundreds of $\mathrm{k}_{\mathrm{B}} \mathrm{T}^{161}$. However, given the solid-like domain formed in charged di-block copolymers, it is tempting to ask how the interleaflet coupling mechanisms would differ for liquid (Lo or Ld) domains, and whether similar PMF calculations would be applicable to investigating cross-layer domain coupling in biological membranes.

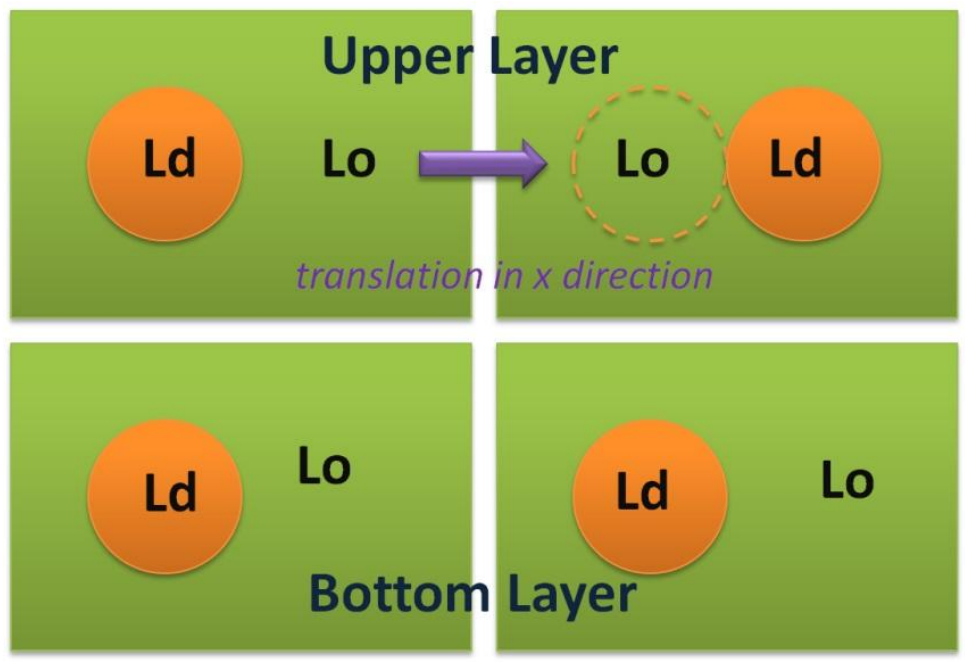

Figure 7. Schematic diagram of two end structures for umbrella sampling simulations of the cross-layer domain coupling in raft-containing lipid bilayers. The system consists of one Ld domain embedded in an Lo phase, with the Lo domains in the two layers being in register in one end structure (left) and not in contact in the other (right).

A detailed PMF calculation on a lipid bilayer model will help resolve the domain coupling mechanism across the chemically asymmetric bilayer leaflets. For example, following the approach of Pantano ${ }^{161}$, a planar bilayer system can be built, consisting of two mixed Lo and Ld phases, with the Lo domains in the two leaflets being in full register in one end structure while completely out of register in the other (Fig. 7). Enhanced sampling techniques, such as umbrella sampling ${ }^{162}$, adaptive biasing force ${ }^{163}$ or steered MD ${ }^{164}$ simulations should be able to be used to compute the PMF profiles for the in-plane translation of the Ld domain from a fully registered state to a completely untouched state. Based on the PMF profiles obtained at different temperatures, the entropic contribution to the domain coupling free energy will be decomposed using the centered difference method ${ }^{150,165}$. Such quantitative MD simulations coupled with further elastic theory analysis should help clarify the roles of line tension, thickness mismatch and acyl chain mixing in the formation of nanoscopic domains, controlling the size of domains and the domain coupling across two bilayer leaflets. 


\section{Mechanisms of leaflet coupling}

Direct measurement of the interleaflet coupling strength of nanoscopic domains still poses a significant experimental challenge. Quantitative estimates of coupling strengths have been obtained from theoretical approaches, including phenomenological modeling 46, 166, 167 and computer simulations ${ }^{156}$. In phenomenological models, the energy of the membrane is described in terms of order parameters, such as composition, curvature and mono-layer thickness. These models provide a useful framework for understanding phase behavior in complex membranes. When coupled with the elastic theory of Helfrich, they have been used to describe the shape of multi-component and multi-phase lipid vesicles. In addition, although limited in their time and length scales, AAMD and CGMD simulations are playing increasingly important roles in providing quantitative and molecular-level insights into interleaflet coupling.

The role of transmembrane proteins Transmembrane proteins are hugely important molecules, interpreting and transducing signals across the bilayer. These molecules are highly evolved to function optimally in the lipid bilayer environment, in this way the bilayer itself serves as an allosteric regulator of protein function. But this is only part of the story; the bilayer also serves to organize multipotent complexes, spanning both leaflets. Additionally, the orientation of transmembrane proteins is sensitive to the compositional asymmetry of the bilayer. The anionic lipids (PS) in the cytoplasmic leaflet associate preferentially with positively charged regions of transmembrane proteins ${ }^{168-170}$. This suggests that lipid organization is either a regulatory tool (lipid raft hypothesis) or an effect of accommodating local protein hydrophobic thickness. In either case, there is a clear role of proteins in coupling of the bilayer leaflets to lateral heterogeneity of lipid composition.

The regulation of transmembrane protein function occurs through a number of parameters, such as bilayer fluidity ${ }^{171}$, bilayer stiffness ${ }^{172}$, intrinsic curvature of the constituent lipids ${ }^{173}$, bilayer compression or stretching due to bilayer/protein hydrophobic mismatch ${ }^{129}$, or lateral pressure/tension effects ${ }^{174}$. Some of these mechanisms are the same as those that impact membrane lateral organization (Fig. 8). Hydrophobic mismatch in particular seems to be important with respect to lateral organization, as even lipid only systems show a remarkable sensitivity to hydrophobic mismatch in phase separated systems ${ }^{136}$. Work with transmembrane peptides further reinforces this notion; fluorescence and detergent resistance studies have found that peptide hydrophobic thickness is a critical parameter for its partitioning between Lo and Ld phases ${ }^{175-179}$. 

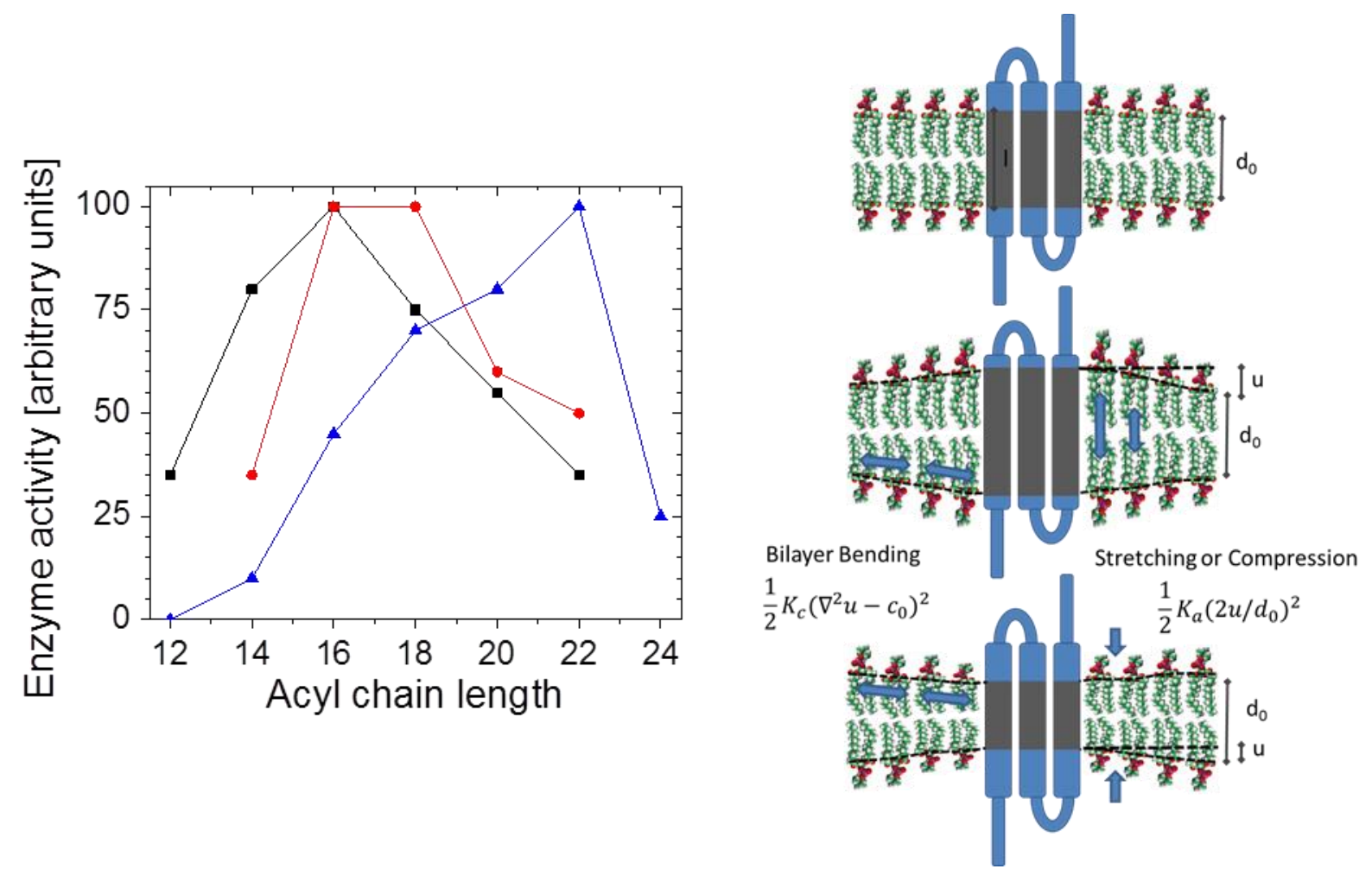

Figure 8. (Left) Transmembrane protein activity is sensitive to hydrophobic thickness of the bilayer: (black) E. coli melibiose-cation contransporter ${ }^{180}$, (red) L. lactis Leu- $\mathrm{H}^{+}$cotransporter ${ }^{181}$ and (blue) sarcoplasmic reticulum $\mathrm{Ca}^{2+}$-ATPase ${ }^{182}$. (Right) Structural distortions in the protein and bilayer structures due to hydrophobic mismatch. The membrane adapts to the hydrophobic thickness of transmembrane proteins by locally stretching or compressing ${ }^{183}$, as well as deformations from bilayer bending ${ }^{173}$. Figure adapted from Andersen and Koeppe ${ }^{184}$.

Electrostatic coupling We have already alluded to the role of charge in the orientation of transmembrane proteins, but bilayer charge is also a consideration in the context of leaflet coupling for pure lipid systems. Cell membranes control many critical biological processes through electrostatic mechanisms, such as the transport of ions, the insertion of membrane proteins and phase separation of lipid and protein-lipid domains. A non-linear Poisson-Boltzmann model has been used to quantitatively describe domain coupling in simplified lipid bilayer models ${ }^{46,185,186}$. This analysis suggests that charged domains would have a small repulsive effect upon each other, favoring domain anti-correlation. This effect is thought to be one to two orders of magnitude weaker than the theoretical estimates of the bilayer mismatch energy from Collins ${ }^{187}\left(0.1-1 \mathrm{k}_{\mathrm{B}} \mathrm{T} / \mathrm{nm}^{2}\right)$ or Risselada and Marrink ${ }^{156}\left(0.1-0.2 \mathrm{k} \mathrm{B} / \mathrm{nm}^{2}\right)$. Although the electrostatic interaction of lipid headgroups in itself cannot be invoked to explain the interleaflet coupling, this does not exclude the possibility that electrostatic interaction acts together with other membrane properties, such as curvature and/or local composition, to have a strong and collective influence on the cross-layer domain coupling.

Cholesterol flip-flop Cholesterol is more mobile in bilayers resembling the Ld phase than those resembling the Lo phase ${ }^{156,158}$. In disordered bilayers, cholesterol rapidly moves from one bilayer leaflet to the other (flip-flop) ${ }^{188}$, fully exploiting the orientational and translational freedom afforded in both leaflets. The flip-flop rate has been observed experimentally, giving a half-life of approximately 1 minute in a given leaflet ${ }^{97,189}$. Recent MD simulations showed rapid cholesterol flip-flop in membranes that mimic the composition of biological membranes, and confirmed that the flip-flop rate is much higher in the Ld phase than in the Lo phase ${ }^{156,190}$. 
These considerations lead to the idea that cholesterol will experience a different degree of confinement in registered domains (with opposing leaflets in the same phases) and in anti-registered domains (with opposing leaflets in different phases), suggesting that the cholesterol flip-flop can potentially be an important factor contributing to domain coupling from an entropic point of view. Based on this consideration, $\mathrm{May}^{46}$ estimated the contribution of the cholesterol flip-flop to the interleaflet coupling. In his model, cholesterol motion is confined in one leaflet for anti-registered domains while cholesterol is free to move in the membrane normal direction for registered domains. This entropic effect will favor alignment of Lo-Lo and Ld-Ld domains instead of mismatched Lo-Ld domains. By only considering the translational entropy and the desolvation of hydroxyl group of cholesterol, the contribution from the cholesterol flip-flop was estimated to be $\sim 0.003 \mathrm{k}_{\mathrm{B}} \mathrm{T} / \mathrm{nm}^{2}{ }^{26}$. This value is, however, more than an order of magnitude smaller than other estimated contributions, such as acyl chain interdigitation and interleaf tensions. This is consistent with the assertion of Putzel ${ }^{166}$, who showed using a mean field model that the cholesterol flip-flop contributes $<1 \%$ to the total interleaflet coupling strength. Therefore, both studies excluded the possibility that cholesterol flip-flop is a main contributing mechanism underlying the interleaflet domain coupling.

Acyl chain interdigitation Dynamic interdigitation of acyl chains into the opposing leaflet provides another mechanism for coupling between domains in the two leaflets. When the terminal segments of the lipid acyl chains cross the bilayer midplane they increase the entropy of the chains and reduce the free energy of the system, as in the case of apposed disordered bilayers ${ }^{143}$ such as an Ld-Ld phase region. However, Lo regions pack more tightly, with the acyl tails of lipids almost fully extended, eliminating the free space needed for the opposing leaflet to interdigitate ${ }^{191}$. Similarly, if an Ld region is aligned opposite to an Lo region, the Lo leaflet will hamper penetration of lipid chains in the Ld phase, thus decreasing the lipid chain entropy and increasing the overall free energy. Based on a mean-field chain packing theory by Szleifer et al ${ }^{192}$, May ${ }^{46}$ estimated the coupling strength of the acyl chain interdigitation to be $\sim 0.1$ $\mathrm{k}_{\mathrm{B}} \mathrm{T} / \mathrm{nm}^{2}$. Putzel et al. ${ }^{166}$ also found that the coupling strength increases as the fraction of saturated lipids is increased, indicating that the saturated lipid chains contribute most to the interdigitation at the bilayer midplane and thus also to the interleaflet coupling.

Chiantia and London have utilized their recently developed liposome-based asymmetric membrane systems to gain a deeper understanding of the influence of acyl chain length and saturation on the interleaflet coupling in asymmetric bilayers ${ }^{30}$. They systematically determined diffusion coefficients (by fluorescence correlation spectroscopy) and order parameters (from fluorescence lifetime) in each individual leaflet of the lipid bilayer independently. Significant interleaflet coupling of lipid diffusion was observed, which depends on both the length and saturation of lipid acyl chains. They postulated that the molecular mechanism for the chain interdigitation is likely to involve van der Waals interactions between the terminal portions of the acyl chains of facing lipids occurring at and near the bilayer midplane. They further suggested that fine tuning of the lipid composition might be used to mediate interleaflet coupling in biological membranes. To elucidate the effects of chain-length mismatch and mono-unsaturation on bilayer structure and dynamics, Niemela et al. performed AAMD simulations of lipid bilayers with a number of structurally different sphingomyelin molecules ${ }^{143}$. In particular, they quantified the extent of the acyl chain interdigitation by plotting electron density and molecular number density profiles, and found that increasing the acyl chain length furthers the interdigitation across the bilayer center. Interdigitation was also found to be stronger for unsaturated than saturated chains ${ }^{143}$. Another promising approach involves the use of the so-called two-phase thermodynamic (2PT) model to quantify the entropy of molecular fluids from MD simulations ${ }^{193}$. Given the liquid-like nature of lipid bilayer cores, the application of the 2PT model will quantify the entropic contribution of dynamic chain interdigitation, thus clarifying its role in the cross-layer domain coupling. 
Composition-curvature coupling It has been shown that certain lipid compositions phase separate into Ld and Lo domains ${ }^{194}$, similar to those predicted for the lipid raft hypothesis ${ }^{1}$. Coupling of curvature, bending, and composition is thought to be one mechanism capable of stabilizing domain sizes at finite values ${ }^{195-197}$. This concept can be extended to asymmetric lipid bilayers and has been considered with a number of phenomenological models ${ }^{46}$. Hansen et al. introduced membrane curvature as a second order parameter (besides composition), where a direct compositional coupling between the two leaflets (e.g., $\gamma \phi_{1} \phi_{2}, \gamma$ is coupling strength, $\phi_{1}$ and $\phi_{2}$ are the compositions of the two leaflets, respectively) was analyzed ${ }^{198}$. Based on the Landau free energy approach, they investigated the interplay between monolayer cooperative phenomena and inter-monolayer coupling within a bilayer. Similarly, Wagner et al. ${ }^{167}$ developed a minimal phenomenological model, which predicted that a monolayer with a propensity for phase separation is able to induce phase separation in the apposed monolayer, while a monolayer without this propensity is able to prevent phase separation in the apposed monolayer. It also predicted that either complete or partial registration of the monolayer domains can occur across the two leaflets depending on the critical coupling strength. Through systematic CGMD simulations, Perlmutter and Sachs found substantial effects of compositional asymmetry on local bilayer curvature, and proposed that the induced curvature may be involved in the interleaflet domain coupling across asymmetric bilayers ${ }^{62}$.

Schick and coworkers developed a statistical mechanical model of coupled fluctuating domain interfaces and used it to clarify the relationship between the interleaflet coupling and the extent of interleaflet alignment of liquid domains ${ }^{166}$. The characteristic size of fluctuations out of domain registry was found to depend only weakly on the strength of the interleaflet coupling, on the order of $\mathrm{nm}$, which raised questions regarding whether it is valid to estimate the coupling strength from CGMD simulations of nanoscopic sized domains based on the analysis of domain overlapping size fluctuations ${ }^{156}$. Finally, recent computational work has isolated the curvature coupling from individual monolayers, to explain the stabilization of membrane domains on nanoscopic length scales ${ }^{199}$. The authors suggested an analogy between the ripple phase seen in single component bilayer models and domain coupling between leaflets.

Line and interleaflet tensions In phase separation models based on compositional ordering, an excess of boundary energy leads to domain coarsening ${ }^{197}$, this excess energy is called the line tension. The reason that this leads to coarsening is that the contribution of line tension to the free energy of the system scales with interfacial length, thus minimizing length reduces the global free energy. The magnitude of the line tension scales with a number of parameters and has been widely studied ${ }^{117,136,200-202}$. A two dimensional analog to this concept is an interleaflet tension, a force scaling with overlap area of domains that either favors or disfavors domain registry based on its sign. Collins ${ }^{187}$ postulated that the two tensions originate from the same nature, i.e., the interactions between hydrocarbon chains, and that domain registry would be governed by the balance of these two tensions. Through this approach, he estimated the domain coupling strength to be $\sim 0.5 \mathrm{k}_{\mathrm{B}} \mathrm{T} / \mathrm{nm}^{2}{ }^{187}$.

Since the magnitude of the interleaflet tension scales with the domain area, while the magnitude of the line tension increases proportionally with the length of the domain boundary, the interleaflet contribution grows more quickly than that of the line tension. Therefore, in the two competing tension theory, for small domains the line tension will dominate, which favors domain anti-registration, while for large domains, the interleaflet tension will dominate, which favors domain registration ${ }^{46,62}$. This explains the presence of registered (micron-sized) domains in fluorescence microscopy experiments and antiregistered nanoscopic or solid domains in simulations ${ }^{62}$ and NMR experiments ${ }^{58,59}$. Both cases are quite different from biological membrane domains that can be as small as tens of $\mathrm{nm}$ and are thought to be in register according to other biochemical assays ${ }^{49,52}$.

\section{Conclusions and perspectives}


Domain-coupling mechanisms have been mostly derived from phenomenological models using meanfield theories. An advantage of the mean field approach is that each of the free energy terms can be separately given and assessed. For example, using an extended Landau theory, Schick and coworkers described the domain coupling mechanisms and estimated the coupling strength ${ }^{166}$. They showed that no single contribution such as configurational entropy or gauche bond energy is wholly responsible for the coupling strength but rather by a complex interplay between these properties. Attempts to describe the interleaflet coupling as a simple mechanism are therefore somewhat unrealistic. Furthermore, free energy functionals in phenomenological models are often constructed ad hoc, based on molecular-level models or on a Landau-like series expansion, thus warranting caution when interpreting interleaflet mechanisms and strength using these models. Small changes in input parameters can have a drastic effect on the magnitude or even the sign of individual contributions to the coupling free energy. Complication also arises from the fact that the mean-field analysis neglects thermal fluctuations, which may bias the predictions of interleaflet coupling in various aspects.

At the molecular level, interface mismatch energy and line tension are a manifestation of the imbalance of intermolecular forces, such as van der Waals, electrostatic, solvation (hydration), and steric experienced by molecules located in and around the phase (domain) boundaries. It is thus tempting to understand how interface mismatch energy and line tension arise from the imbalance of these intermolecular forces. CG or atomistic MD simulations provide a link between molecular-level interactions and the mean-field theory. Given recent advances in computer hardware and software, MD simulations are especially likely to play an increasingly important role in elucidating the coupling mechanisms and strength of membrane domains across two opposing leaflets.

A clear path forward will be to study the lipid-protein interactions that regulate nanoscale raft protein assemblies, and how they coalesce to form functional domains. Simultaneously, additional work looking at pure lipid systems will be informative in validating and quantifying the coupling mechanism that have been put forward to date. These studies will rely on new model systems and advances in experimental techniques. The strengths of neutron scattering in particular will be a vital tool in disentangling the role of asymmetry.

Domain coupling between the inner and outer leaflets of a cell has significant implication for biological functions. For example, G-protein-coupled receptors (GPCRs) are involved in numerous signal transduction pathways in response to the presence (binding) of hormones, neurotransmitters and pharmacologic agonists. For efficient and controllable signal transduction, the interaction and thus localization of receptors and post-receptor components in subcellular compartments needs to be coordinated. Induction of domains across leaflets of cell membranes provides a platform for colocalization of signaling components, allowing cells to tailor their responses by spatially organizing the molecules involved in GPCR signal transduction ${ }^{203,204}$. In addition to a demonstrated role in cellular signal transduction, domain coupling may also play important roles in microbial pathogen and toxin entry into host cells, such as HIV virus, influenza virus, measles virus and cholera toxin ${ }^{205}$. Although many biological functions take place in membranes where proteins and lipids are in intimate contact, an understanding of how lipids and proteins function together has been lacking ${ }^{206}$. This simply reinforces the perspective that biological systems utilize a range of mechanisms in concert in order to regulate the physical state of the membrane.

\section{Acknowledgments}

JDN is partially supported by the U.S. DOE BES through the EPSCoR Grant No. DE-FG02-08ER46528. $\mathrm{XC}$ is partially supported by the Laboratory Directed R\&D (LDRD) fund P7394 at the Oak Ridge National Laboratory. This research used resources of the Oak Ridge Leadership Computing Facility at the Oak Ridge National Laboratory, which is supported by the Office of Science of the U.S. Department of Energy under Contract No. DE-AC05-00OR22725. 


\section{References}

1. $\quad$ K. Simons and E. Ikonen, Nature 387 (6633), 569-572 (1997).

2. K. Simons and D. Toomre, Nature reviews Molecular cell biology 1 (1), 31-39 (2000).

3. C. Gajate and F. Mollinedo, Blood 98 (13), 3860-3863 (2001).

4. M. A. del Pozo, N. B. Alderson, W. B. Kiosses, H.-H. Chiang, R. G. Anderson and M. A. Schwartz, Science 303 (5659), 839-842 (2004).

5. $\quad$ S. K. Pierce, Nature Reviews Immunology 2 (2), 96-105 (2002).

6. H. Hering, C.-C. Lin and M. Sheng, The Journal of neuroscience 23 (8), 3262-3271 (2003).

7. M. Villalba, K. Bi, F. Rodriguez, Y. Tanaka, S. Schoenberger and A. Altman, The Journal of cell biology 155 (3), 331-338 (2001).

8. $\quad$ Lingwood D and Simons K, Science 327 (5961), 46-50 (2010).

9. Jacobson K, Mouritsen OG and Anderson RG, Nat Cell Biol 9 (1), 7-14 (2007).

10. A. Pralle, P. Keller, E.-L. Florin, K. Simons and J. Hörber, The Journal of cell biology 148 (5), 997-1008 (2000).

11. L. Pike, Biochem. J 378, 281-292 (2004).

12. A. V. Samsonov, I. Mihalyov and F. S. Cohen, Biophysical journal 81 (3), 1486-1500 (2001).

13. D. A. Brown and E. London, Journal of Biological Chemistry 275 (23), 17221-17224 (2000).

14. A. Verkleij, R. Zwaal, B. Roelofsen, P. Comfurius, D. Kastelijn and L. Van Deenen, Biochimica et Biophysica Acta (BBA)-Biomembranes 323 (2), 178-193 (1973).

15. J. A. Allen, R. A. Halverson-Tamboli and M. M. Rasenick, Nature Reviews Neuroscience 8 (2), 128-140 (2007).

16. S. Mongrand, J. Morel, J. Laroche, S. Claverol, J.-P. Carde, M.-A. Hartmann, M. Bonneu, F. Simon-Plas, R. Lessire and J.-J. Bessoule, Journal of Biological Chemistry 279 (35), 3627736286 (2004).

17. D. López and R. Kolter, Genes \& development 24 (17), 1893-1902 (2010).

18. F. G. van der Goot and T. Harder, presented at the Seminars in immunology, 2001 (unpublished).

19. R. A. Dick, S. L. Goh, G. W. Feigenson and V. M. Vogt, Proceedings of the National Academy of Sciences 109 (46), 18761-18766 (2012).

20. G. Van Meer, D. R. Voelker and G. W. Feigenson, Nature reviews molecular cell biology 9 (2), 112-124 (2008).

21. M. S. Bretscher, Nature 236 (61), 11-12 (1972).

22. M. S. Bretscher, Science 181 (4100), 622-629 (1973).

23. T. L. Steck and G. Dawson, Journal of biological chemistry 249 (7), 2135-2142 (1974).

24. J. Rothman and J. Lenard, Science 195 (4280), 743-753 (1977).

25. R. F. A. Zwaal, P. Comfurius and L. L. M. Van Deenen, Nature 268 (5618), 358-360 (1977).

26. J. A. Op den Kamp, Annual review of biochemistry 48 (1), 47-71 (1979).

27. S. Singer and G. L. Nicolson, Day and Good Membranes and viruses in immunopathology, 7-47 (1972). 
28. W. Gibson Wood, U. Igbavboa, W. E. Müller and G. P. Eckert, Journal of Neurochemistry 116 (5), 684-689 (2011).

29. Cheng HT, Megha and London E, J Biol Chem 284 (10), 6079-6092 (2009).

30. Chiantia S and London E, Biophys J 103 (11), 2311-2319 (2012).

31. P. F. Devaux, Biochemistry 30 (5), 1163-1173 (1991).

32. V. A. Fadok, D. R. Voelker, P. A. Campbell, J. J. Cohen, D. L. Bratton and P. M. Henson, The Journal of Immunology 148 (7), 2207-2216 (1992).

33. S. Martin, C. Reutelingsperger, A. J. McGahon, J. A. Rader, R. Van Schie, D. M. LaFace and D. R. Green, The Journal of experimental medicine 182 (5), 1545-1556 (1995).

34. M. Bennett, D. Gibson, S. Schwartz and J. Tait, Circulation research 77 (6), 11361142 (1995).

35. L. Casciola-Rosen, A. Rosen, M. Petri and M. Schlissel, Proceedings of the National Academy of Sciences 93 (4), 1624-1629 (1996).

36. E. M. Bevers, P. Comfurius, J. Vanrijn, H. C. Hemker and R. F. A. Zwaal, Eur. J. Biochem. 122 (2), 429-436 (1982).

37. Y. Tanaka and A. Schroit, Journal of Biological Chemistry 258 (18), 11335-11343 (1983).

38. A. J. Schroit, J. W. Madsen and Y. Tanaka, Journal of Biological Chemistry 260 (8), 5131-5138 (1985).

39. V. A. Fadok, D. J. Laszlo, P. W. Noble, L. Weinstein, D. Riches and P. Henson, The Journal of Immunology 151 (8), 4274-4285 (1993).

40. J. Connor, C. Bucana, I. J. Fidler and A. J. Schroit, Proceedings of the National Academy of Sciences 86 (9), 3184-3188 (1989).

41. T. Utsugi, A. J. Schroit, J. Connor, C. D. Bucana and I. J. Fidler, Cancer Research $\mathbf{5 1}$ (11), 3062-3066 (1991).

42. M. Seigneuret and P. F. Devaux, Proceedings of the National Academy of Sciences $\mathbf{8 1}$ (12), 3751-3755 (1984).

43. E. M. Bevers, P. Comfurius, D. W. Dekkers and R. F. Zwaal, Biochimica et Biophysica Acta (BBA)-Molecular and Cell Biology of Lipids 1439 (3), 317-330 (1999).

44. Kiessling V, Wan C and Tamm LK, Biochim Biophys Acta 1788 (1), 64-71 (2009).

45. Devaux PF and Morris R, Traffic 5 (4), 241-246 (2004).

46. May S, Soft Matter 5, 3148-3156 (2009).

47. D. Marquardt, B. Geier and G. Pabst, Membranes 5 (2), 180 (2015).

48. V. Kiessling, C. Wan and L. K. Tamm, Biochimica et Biophysica Acta (BBA)Biomembranes 1788 (1), 64-71 (2009).

49. Harder T, Scheiffele P, Verkade P and Simons K, J Cell Biol 141 (4), 929-942 (1998).

50. T.-Y. Wang and J. R. Silvius, Biophysical journal 84 (1), 367-378 (2003).

51. Kiessling V, Crane JM and Tamm LK, Biophys J 91 (9), 3313-3326 (2006).

52. Zacharias DA, Violin JD, Newton AC and Tsien RY, Science 296 (5569), 913-916 (2002).

53. J. D. C. Nickels, X.; Mostofian, B.; Lindner, B., Heberle, F. A.; Perticaroli, S.; Stanley, C.; Feygenson, Smith, J.C.; Egami, T.; Standaert, R. F.; Myles, D. A. A.; Ohl, M.; Katsaras, J., (2015).

54. C. Dietrich, L. Bagatolli, Z. Volovyk, N. Thompson, M. Levi, K. Jacobson and E. Gratton, Biophysical journal 80 (3), 1417-1428 (2001). 
55. S. Garg, J. Rühe, K. Lüdtke, R. Jordan and C. A. Naumann, Biophysical journal 92 (4), 1263-1270 (2007).

56. Collins MD and Keller SL, Proc Natl Acad Sci U S A 105 (1), 124-128 (2008).

57. Korlach J, Schwille P, Webb WW and Feigenson GW, Proc Natl Acad Sci U S A 96 (15), 8461-8466 (1999).

58. Zhang J, Jing B, Tokutake N and Regen SL, J Am Chem Soc 126 (35), 10856-10857 (2004).

59. Almeida PF, Vaz WL and Thompson TE, Biochemistry 31 (7198-210) (1992).

60. Khanna K, Chang TT and Kindt JT, J Chem Phys 124 (3), 036102 (2006).

61. Stevens MJ, J Am Chem Soc 127 (44), 15330-15331 (2005).

62. Perlmutter JD and Sachs JN, J Am Chem Soc 133, 6563-6577 (2011).

63. Cheng HT and London E, Biophys J 100 (11), 2671-2678 (2011).

64. M. H. Tamm LK, Biophys J 47 (1), 105-113 (1985).

65. J. M. Crane, V. Kiessling and L. K. Tamm, Langmuir 21 (4), 1377-1388 (2005).

66. T. Baumgart and A. Offenhäusser, Langmuir 19 (5), 1730-1737 (2003).

67. I. Visco, S. Chiantia and P. Schwille, Langmuir 30 (25), 7475-7484 (2014).

$68 . \quad$ B. L. Stottrup, S. L. Veatch and S. L. Keller, Biophysical journal 86 (5), 2942-2950 (2004).

69. G. Gregoriadis and D. E. Neerunjun, Eur. J. Biochem. 47 (1), 179-185 (1974).

70. D. Papahadjopoulos, T. M. Allen, A. Gabizon, E. Mayhew, K. Matthay, S. K. Huang, K. D. Lee, M. C. Woodle, D. D. Lasic and C. Redemann, Proceedings of the National Academy of Sciences 88 (24), 11460-11464 (1991).

71. R. Langer, Science 249 (4976), 1527-1533 (1990).

72. M. L. Immordino, F. Dosio and L. Cattel, International journal of nanomedicine 1 (3), 297 (2006).

73. L. Djordjevich and I. Miller, Experimental hematology 8 (5), 584-592 (1980).

74. J. Nickels and A. F. Palmer, Langmuir 19 (25), 10581-10587 (2003).

75. A. F. Palmer, P. Wingert and J. Nickels, Biophysical Journal 85 (2), 1233-1247 (2003).

76. S. L. Li, J. Nickels and A. F. Palmer, Biomaterials 26 (17), 3759-3769 (2005).

77. M. Baptist, C. Panagabko, J. Nickels, J. Katsaras and J. Atkinson, Lipids 50 (3), 323328 (2015).

78. H. T. McMahon, M. M. Kozlov and S. Martens, Cell 140 (5), 601-605 (2010).

79. B. Różycki and R. Lipowsky, The Journal of chemical physics 142 (5), 054101 (2015).

80. Z. Huang and E. London, Langmuir 29 (47), 14631-14638 (2013).

81. Lin Q and London E, PLoS One 9 (1), e87903 (2014).

82. M. Son and E. London, Journal of lipid research 54 (12), 3385-3393 (2013).

83. M. Son and E. London, Journal of lipid research 54 (1), 223-231 (2013).

84. G. Vladisavljević, I. Kobayashi and M. Nakajima, Microfluidics and nanofluidics $\mathbf{1 3}$ (1), 151-178 (2012).

85. S. Pautot, B. J. Frisken and D. Weitz, Proceedings of the National Academy of Sciences 100 (19), 10718-10721 (2003).

86. H. C. Shum, D. Lee, I. Yoon, T. Kodger and D. A. Weitz, Langmuir 24 (15), 7651-7653 (2008). 
87. K. Funakoshi, H. Suzuki and S. Takeuchi, Analytical chemistry 78 (24), 8169-8174 (2006).

88. K. Funakoshi, H. Suzuki and S. Takeuchi, Journal of the American Chemical Society 129 (42), 12608-12609 (2007).

89. P. C. Hu, S. Li and N. Malmstadt, ACS Applied Materials \& Interfaces 3 (5), 1434-1440 (2011).

90. D. L. Richmond, E. M. Schmid, S. Martens, J. C. Stachowiak, N. Liska and D. A. Fletcher, Proceedings of the National Academy of Sciences 108 (23), 9431-9436 (2011).

91. T. Hamada, Y. Miura, Y. Komatsu, Y. Kishimoto, M. d. Vestergaard and M. Takagi, The Journal of Physical Chemistry B 112 (47), 14678-14681 (2008).

92. H. M. McConnell and R. D. Kornberg, Biochemistry 10 (7), 1111-1120 (1971).

93. J. Liu and J. C. Conboy, Biophysical journal 89 (4), 2522-2532 (2005).

94. K. John, S. Schreiber, J. Kubelt, A. Herrmann and P. Müller, Biophysical journal 83 (6), 3315-3323 (2002).

95. M. Nakano, M. Fukuda, T. Kudo, N. Matsuzaki, T. Azuma, K. Sekine, H. Endo and T. Handa, The Journal of Physical Chemistry B 113 (19), 6745-6748 (2009).

96. S. Garg, L. Porcar, A. Woodka, P. Butler and U. Perez-Salas, Biophysical journal 101 (2), 370-377 (2011).

97. R. Leventis and J. R. Silvius, Biophysical journal 81 (4), 2257-2267 (2001).

98. G. van Meer, Cold Spring Harbor perspectives in biology 3 (5), a004671 (2011).

99. T. Lhermusier, H. Chap and B. Payrastre, Journal of Thrombosis and Haemostasis 9 (10), 1883-1891 (2011).

100. Noireaux V, Maeda YT and Libchaber A, Proc Natl Acad Sci U S A 108 (9), 3473-3480 (2011).

101. Daleke DL, J Biol Chem 282, 821-825 (2007).

102. Kim DM and Swartz JR, Biotechnol Bioeng 66, 180-188 (1999).

103. J. C. McIntyre and R. G. Sleight, Biochemistry 30 (51), 11819-11827 (1991).

104. K. Kinosita, S. Kawato and A. Ikegami, Biophysical journal 20 (3), 289-305 (1977).

105. D. Marsh, in Membrane spectroscopy (Springer, 1981), pp. 51-142.

106. W.-C. Lin, C. D. Blanchette, T. V. Ratto and M. L. Longo, Biophysical journal 90 (1), 228-237 (2006).

107. J. Yuan, C. Hao, M. Chen, P. Berini and S. Zou, Langmuir 29 (1), 221-227 (2012).

108. C. Wan, V. Kiessling and L. K. Tamm, Biochemistry 47 (7), 2190-2198 (2008).

109. S. Chiantia, P. Schwille, A. S. Klymchenko and E. London, Biophysical journal 100 (1), L1-L3 (2011).

110. D. Lingwood and K. Simons, Nature protocols 2 (9), 2159-2165 (2007).

111. R. Zidovetzki and I. Levitan, Biochimica et Biophysica Acta (BBA)-Biomembranes 1768 (6), 1311-1324 (2007).

112. Yuan C, Furlong J, Burgos P and Johnston LJ, Biophys J 82 (5), 2526-2535 (2002).

113. Johnston LJ, Langmuir 23 (11), 5886-5895 (2007).

114. F. Tokumasu, A. J. Jin, G. W. Feigenson and J. A. Dvorak, Biophysical journal 84 (4), 2609-2618 (2003).

115. Stöckl MT and Herrmann A, Biochim Biophys Acta 1798 (7), 1444-1456 (2010).

116. Goksu EI, Vanegas JM, Blanchette CD, Lin WC and Longo ML, Biochim Biophys Acta 1788 (1), 254-266 (2009).

117. T. Baumgart, S. T. Hess and W. W. Webb, Nature 425 (6960), 821-824 (2003). 
118. Klymchenko AS and Kreder R, Chem Biol 21 (1), 97-113 (2014).

119. G. J. Schütz, G. Kada, V. P. Pastushenko and H. Schindler, The EMBO journal 19 (5), 892-901 (2000).

120. L. Bagatolli and E. Gratton, Biophysical journal 78 (1), 290-305 (2000).

121. K. Simons and M. J. Gerl, Nature reviews Molecular cell biology 11 (10), 688-699 (2010).

122. E. A. Jares-Erijman and T. M. Jovin, Nature biotechnology 21 (11), 1387-1395 (2003).

123. R. F. de Almeida, L. M. Loura, A. Fedorov and M. Prieto, Journal of molecular biology 346 (4), 1109-1120 (2005).

124. G. W. Feigenson, Biochimica et Biophysica Acta (BBA)-Biomembranes 1788 (1), 47$52(2009)$.

125. A. K. Kenworthy, N. Petranova and M. Edidin, Molecular Biology of the Cell 11 (5), 1645-1655 (2000).

126. Sezgin E, Levental I, Grzybek M, Schwarzmann G, Mueller V, Honigmann A and Belo VN, Biochimica et Biophysica Acta 1818 (7), 1777-1784 (2012).

127. E. Wu, K. Jacobson and D. Papahadjopoulos, Biochemistry 16 (17), 3936-3941 (1977).

128. Z. Derzko and K. Jacobson, Biochemistry 19 (26), 6050-6057 (1980).

129. O. G. Mouritsen and K. Jørgensen, Chemistry and physics of lipids 73 (1), 3-25 (1994).

130. P. F. Almeida, W. L. Vaz and T. Thompson, Biochemistry 31 (31), 7198-7210 (1992).

131. G. Büldt, H. Gally, A. Seelig, J. Seelig and G. Zaccai, (1978).

132. G. Büldt, H. Gally, J. Seelig and G. Zaccai, Journal of molecular biology 134 (4), 673691 (1979).

133. G. Zaccai, G. Büldt, A. Seelig and J. Seelig, Journal of molecular biology 134 (4), 693706 (1979).

134. D. I. Svergun, Biophysical Journal 76 (6), 2879-2886 (1999).

135. Pencer J, Mills T, Anghel V, Krueger S, Epand RM and Katsaras J, Eur Phys J E 18, 447-458 (2005).

136. Heberle FA, Petruzielo RS, Pan J, Drazba P, Kučerka N, Standaert RF, Feigenson GW and Katsaras J, J Am Chem Soc 135 (18), 6853-6859 (2013).

137. S. Qian and W. T. Heller, The Journal of Physical Chemistry B 115 (32), 9831-9837 (2011).

138. A. Zilman and R. Granek, Physical Review Letters 77 (23), 4788 (1996).

139. G. I. King and S. H. White, Biophysical Journal 49 (5), 1047-1054 (1986).

140. S. H. White and G. I. King, Proceedings of the National Academy of Sciences 82 (19), 6532-6536 (1985).

141. N. Kučerka, J. F. Nagle, S. E. Feller and P. Balgavý, Physical Review E 69 (5), 051903 (2004).

142. T. Gulik-Krzywicki, E. Rivas and V. Luzzati, Journal of Molecular Biology 27 (2), 303322 (1967).

143. Niemelä PS, Hyvönen MT and Vattulainen I, Biophys J 90 (3), 851-863 (2006).

144. Sodt AJ, Sandar ML, Gawrisch K, Pastor RW and Lyman E, J Am Chem Soc 136 (2), 725-732 (2014). 
145. Levine ZA, Venable RM, Watson MC, Lerner MG, Shea JE, Pastor RW and Brown FL, J Am Chem Soc 136 (39), 13582-13585 (2014).

146. Pan J, Cheng X, Heberle FA, Mostofian B, Kučerka N, Drazba P and Katsaras J, J Phys Chem B 116 (51), 14829-14838 (2012).

147. Pan J, Cheng X, Sharp M, Ho CS, Khadka N and Katsaras J, Soft Matter 11((1), 130138 (2015).

148. Cerezo J, Zu'ñiga J, Bastida A, Requena A and Cero'n-Carrascob JP, Phys Chem Chem Phys 15, 6527-6538 (2013).

149. Khadka NK, Cheng X, Ho CS, Katsaras J and Pan J, Biophys J 108, 1-10 (2015 ).

150. Bennett WF, MacCallum JL, Hinner MJ, Marrink SJ and T. DP., J Am Chem Soc 131 (35), 12714-12720 (2009).

151. E W and Vanden-Eijnden E, Annu Rev Phys Chem 61, 391-420 (2010).

152. Jo S, Rui H, Lim JB, Klauda JB and Im W, J Phys Chem B 114 (42), 13342-13348 (2010).

153. Hong C, Tieleman DP and Wang Y, Langmuir 30 (40), 11993-12001 (2014).

154. Wang Y, Markwick PR, de Oliveira CA and McCammon JA, J Chem Theory Comput 7 (10), 3199-3207 (2011).

155. Marrink SJ, Risselada HJ, Yefimov S, Tieleman DP and de Vries AH, J Phys Chem B 111 (27), 7812-7824 (2007).

156. Risselada HJ and Marrink SJ, Proc Natl Acad Sci USA 105 (45), 17367-17372 (2008).

157. Ingólfsson HI, Melo MN, van Eerden FJ, Arnarez C, Lopez CA, Wassenaar TA, Periole X, de Vries AH, Tieleman DP and Marrink SJ, J Am Chem Soc 136 (41), 14554-14559 (2014). 158. Marrink SJ, de Vries AH, Harroun TA, Katsaras J and Wassall SR, J Am Chem Soc 130 (1), 10-11 (2008).

159. Pantano DA, Klein ML, Discher DE and Moore PB, J Phys Chem B 115 (16), 46894695 (2011).

160. Jarzynski C, Phys Rev Lett 78 (2690) (1997).

161. Pantano DA, Moore PB, Kleinc ML and Dischera DE, Soft Matter 7, 8182-8191 (2011).

162. Torrie GM and Valleau JP, J Comput Phys 23 (2), 187-199 (1977, Pages ).

163. Darve E, Rodríguez-Gómez D and Pohorille A, J Chem Phys 128 (14), 144120 (2008).

164. Park S, Khalili-Araghi F, Tajkhorshid E and Schulten K, J Chem Phys 119, 3559-3566 (2003).

165. Kubo MM, Gallicchio E and Levy RM, J Phys Chem B 101, 10527-10534 (1997).

166. Putzel GG, Uline MJ, Szleifer I and Schick M, Biophys J 100 (4), 996-1004 (2011).

167. Wagner AJ, Loew S and May S, Biophys J 93 (12), 4268-4277 (2007).

168. Q. Lin and E. London, Journal of Biological Chemistry 289 (9), 5467-5478 (2014).

169. G. HEIJNE and Y. GAVEL, Eur. J. Biochem. 174 (4), 671-678 (1988).

170. G. Gafvelin, M. Sakaguchi, H. Andersson and G. von Heijne, Journal of Biological Chemistry 272 (10), 6119-6127 (1997).

171. M. P. Sheetz and S. J. Singer, Proceedings of the National Academy of Sciences $\mathbf{7 1}$ (11), 4457-4461 (1974).

172. J. Lundbaek, P. Birn, S. Tape, G. E. Toombes, R. Søgaard, R. E. Koeppe, S. M. Gruner, A. J. Hansen and O. S. Andersen, Molecular pharmacology 68 (3), 680-689 (2005).

173. S. M. Gruner, Proceedings of the National Academy of Sciences 82 (11), 3665-3669 (1985). 
174. R. S. Cantor, Biochemistry 36 (9), 2339-2344 (1997).

175. M. R. de Planque and J. A. Killian*, Molecular membrane biology 20 (4), 271-284 (2003).

176. B. Y. van Duyl, D. T. Rijkers, B. de Kruijff and J. A. Killian, FEBS letters 523 (1), 79-84 (2002).

177. J. A. Killian and T. K. Nyholm, Current opinion in structural biology 16 (4), 473-479 (2006).

178. T. J. McIntosh, A. Vidal and S. A. Simon, Biophysical journal 85 (3), 1656-1666 (2003).

179. L. V. Schäfer, D. H. de Jong, A. Holt, A. J. Rzepiela, A. H. de Vries, B. Poolman, J. A. Killian and S. J. Marrink, Proceedings of the National Academy of Sciences 108 (4), 13431348 (2011).

180. F. Dumas, J.-F. Tocanne, G. Leblanc and M.-C. Lebrun, Biochemistry 39 (16), 48464854 (2000).

181. G. In't Veld, A. J. Driessen, J. A. O. den Kamp and W. N. Konings, Biochimica et Biophysica Acta (BBA)-Biomembranes 1065 (2), 203-212 (1991).

182. M. Caffrey and G. W. Feigenson, Biochemistry 20 (7), 1949-1961 (1981).

183. O. G. Mouritsen and M. Bloom, Biophysical journal 46 (2), 141-153 (1984).

184. O. S. Andersen and R. E. Koeppe, Annu. Rev. Biophys. Biomol. Struct. 36, 107-130 (2007).

185. C. L. Baciu and S. May, Journal of Physics: Condensed Matter 16 (26), S2455 (2004).

186. A. J. Wagner and S. May, European Biophysics Journal 36 (4-5), 293-303 (2007).

187. Collins MD, Biophys J 94 (5), L32-34 (2008).

188. J. A. Hamilton, Current opinion in lipidology 14 (3), 263-271 (2003).

189. J. M. Backer and E. A. Dawidowicz, Journal of Biological Chemistry 256 (2), 586-588 (1981).

190. Bennett WFD and Tieleman DP, Biochimica et Biophysica Acta (BBA) Biomembranes 1828 (8), 1765-1776 (2013).

191. X. Xu and E. London, Biochemistry 39 (5), 843-849 (2000).

192. Szleifer I, Ben-Shaul A and Gelbart WM, J Phys Chem 94 (12), 5081-5089 (1990).

193. Lin ST, Maiti PK and Goddard WA, J Phys Chem B 114 (24), 8191-8198 (2010).

194. G. W. Feigenson, Biochimica et Biophysica Acta (BBA) - Biomembranes 1788 (1), 47-52 (2009).

195. T. Kawakatsu, D. Andelman, K. Kawasaki and T. Taniguchi, Journal de Physique II 3 (7), 971-997 (1993).

196. T. Taniguchi, K. Kawasaki, D. Andelman and T. Kawakatsu, Journal de Physique II 4 (8), 1333-1362 (1994).

197. M. Seul and D. Andelman, Science 267 (5197), 476-483 (1995).

198. Hansen PL, Miao L and Ipsen JH, Physical Review E 58 (2), 2311-2324 (1998).

199. S. Meinhardt, R. L. Vink and F. Schmid, Proceedings of the National Academy of Sciences 110 (12), 4476-4481 (2013).

200. A. J. García-Sáez, S. Chiantia and P. Schwille, Journal of Biological Chemistry 282 (46), 33537-33544 (2007).

201. P. I. Kuzmin, S. A. Akimov, Y. A. Chizmadzhev, J. Zimmerberg and F. S. Cohen, Biophysical journal 88 (2), 1120-1133 (2005). 
202. A. R. Honerkamp-Smith, P. Cicuta, M. D. Collins, S. L. Veatch, M. den Nijs, M. Schick and S. L. Keller, Biophysical journal 95 (1), 236-246 (2008).

203. O. Soubias and K. Gawrisch, Biochimica et Biophysica Acta (BBA)-Biomembranes 1818 (2), 234-240 (2012).

204. Ostrom RS and Insel PA, Br J Pharmacol 143 (2), 235-245 (2004).

205. Campbell SM, Crowe SM and Mak J, J Clin Virol 22 (3), 217-227 (2001).

206. Kusumi A, Fujiwara TK, Chadda R, Xie M, Tsunoyama TA, Kalay Z, Kasai RS and Suzuki KG, Annu Rev Cell Dev Biol 28, 215-250 (2012).

207. M. Weik, H. Patzelt, G. Zaccai and D. Oesterhelt, Molecular cell 1 (3), 411-419 (1998). 